\title{
Relación entre hábitos de estudio y motivación para el aprendizaje con el rendimiento académico en estudiantes de medicina veterinaria
}

\author{
Relationship between study habits and motivation for learning with academic performance in \\ students of veterinary medicine
}

Graciela Inés Yamada Abe', Víctor Hernán Bazán Rodríguez', Juan Antonio Espinoza Blanco

\section{RESUMEN}

Objetivo: Determinar la relación entre los hábitos de estudio y motivación para el aprendizaje con el rendimiento académico de los estudiantes de medicina veterinaria de la Universidad Nacional Mayor de San Marcos en el año 2014. Métodos: se utilizó como instrumento el cuestionario de hábitos de estudio y motivación para el aprendizaje validado y adaptado en su contenido a la población en estudio, los datos de rendimiento académico fueron los promedios ponderados de los estudiantes, la muestra no probabilística fue de 163 alumnos del segundo al sexto año de estudios, la muestra es aleatoria con $5 \%$ de nivel de significación. Resultados: Determinan que no hay asociación entre las variables y se concluye que no existe relación entre las variables bajo estudio. Al aplicar el análisis de correspondencias múltiples se obtuvo que los estudiantes de 30 años a más; presentan motivación para el aprendizaje óptima, los estudiantes con rendimiento académico regular tienen hábitos de estudio bueno y motivación para el aprendizaje bueno, los estudiantes con rendimiento académico bajo presentan hábitos de estudio regular y motivación para el aprendizaje regular. Conclusiones: El rendimiento académico presenta baja relación lineal frente a los hábitos de estudio y la motivación para el aprendizaje.

Palabras clave: Hábitos de estudio, motivación para el aprendizaje, rendimiento académico, medicina veterinaria.

\section{ABSTRACT}

Objective: To determine the relationship between study habits and motivation for learning with the academic performance of veterinary medicine students of the National University of San Marcos in 2014. Methods: the study habits questionnaire was used as an instrument and motivation for learning validated and adapted in its content to the population under study, the data of academic performance were the weighted averages of the students, the non-probabilistic sample was 163 students from the second to the sixth year of studies, the sample is random with $5 \%$ level of significance. Results: They determine that there is no association between the variables and it is concluded that there is no relationship between the variables under study. When applying the multiple correspondence analysis, it was obtained that students aged 30 years and over; they present motivation for optimal learning, students with regular academic performance have good study habits and motivation for good learning, students with low academic performance have regular study habits and motivation for regular learning. Conclusions: Academic performance has a low linear relationship with study habits and motivation for learning

Keywords: Study habits, motivation for learning, academic performance, veterinary medicine.

${ }^{1}$ Universidad Nacional Mayor de San Marcos. Lima, Perú. 


\section{INTRODUCCIÓN}

Docentes y autoridades toman poco tiempo en evaluar si el graduado en medicina veterinaria reúne las características profesionales, los conocimientos y las habilidades que se busca inculcarle (San Martín y García, 2002). Tomando como ejemplo la Facultad de Medicina Veterinaria de la Universidad Nacional Mayor de San Marcos, de cada 100 ingresantes sólo 36 estudiantes terminan la carrera en los 6 años de estudios (San Martín y García, 2002), por ello debemos tomar en cuenta la cantidad considerable de estudiantes que quedan largo tiempo en el sistema aumentando su estadía en la universidad. Diversas investigaciones realizadas sobre las causas de bajo desempeño académico obtenido por los alumnos ingresantes a la universidad dan cuenta de los factores de mayor incidencia, entre otros, la falta de hábitos de estudio y de motivación, la diferencia de niveles cognoscitivos y las actitudes de los estudiantes, por ello la institución universitaria debe cuestionarse sobre su eficacia para retener y promover estudiantes, así como su función como prestadora de un servicio educativo de calidad. (Pola, 2013).

En nuestro país existen pocas investigaciones relacionadas a la educación veterinaria, por ello queremos conocer la situación real de los alumnos de medicina veterinaria en el Perú tomando como modelo de trabajo a la Facultad de Medicina Veterinaria de la Universidad Nacional Mayor de San Marcos para determinar si el rendimiento académico de los alumnos está relacionado con los hábitos de estudio y la motivación para el aprendizaje.

Desde el punto de vista teórico, utilizar la psicología educativa y la neurociencia para explicar la conducta del estudiante frente al aprendizaje y el logro de objetivos. Desde el punto de vista práctico, realizar un diagnóstico de la situación de los estudiantes de medicina veterinaria en relación a los hábitos de estudio y motivación para el aprendizaje, luego, plantear alternativas de cambio en la manera de estudiar y aprender de nuestros estudiantes para mejorar su rendimiento académico y lograr las competencias deseadas en la educación veterinaria. Desde el punto de vista metodológico, se pretende validar un cuestionario sobre hábitos de estudio y motivación para el aprendizaje acorde a la realidad de nuestro país. Desde el punto de vista social, un estudiante con un buen aprendizaje será un médico veterinario integral con habilidades y conocimientos adecuados para desempeñarse en las áreas de salud animal, salud pública, producción animal e inspección y calidad de los alimentos que contribuirá al desarrollo de nuestra sociedad.

La hipótesis plantea que existe relación entre hábitos de estudio y motivación para el aprendizaje con el rendimiento académico de los estudiantes de Medicina Veterinaria.

El objetivo es determinar la relación entre hábitos de estudio y motivación para el aprendizaje con el rendimiento académico de los estudiantes de Medicina Veterinaria.

\section{MATERIAL Y MÉTODOS}

La investigación es transversal, no experimental y cuantitativa. Se empleó la encuesta para la toma de datos, utilizando como instrumento de medición el Cuestionario de Hábitos de Estudio y Motivación para el Aprendizaje Oñate (1991), que fue validado mediante juicio de expertos donde participaron 10 docentes de la UNMSM y luego fue adaptado al contexto de la población bajo estudio, el cuestionario tiene como objetivo medir y diagnosticar los hábitos de estudio y la motivación para el aprendizaje en estudiantes universitarios.

La población estuvo compuesta por 287 estudiantes de la Facultad de Medicina Veterinaria de la UNMSM matriculados en el año académico 2014, como criterio de exclusión se determinó que los alumnos ingresantes (primer año) no se consideren en el estudio porque aún no han experimentado la dinámica de los estudios universitarios. 
El diseño de la muestra es no probabilístico, estratificado por año de estudios, se consideró como muestra a todos los estudiantes presentes el día de la aplicación del cuestionario y que aceptaron voluntariamente participar en la investigación, haciendo un total de 163.

El instrumento de recolección de datos es el cuestionario que contiene las variables de la investigación operacionalizadas. Para la adaptación se consideró la estructura de las siete dimensiones o factores del cuestionario sobre hábitos de estudio y motivación para el aprendizaje. El cuestionario consta de 2 partes, una relacionada a los hábitos de estudio (52 ítems) y otra sobre la motivación para el aprendizaje (25 ítems).

Las dimensiones o factores sobre hábitos de estudio son: ambientales del estudio, salud física y emocional, aspectos sobre el método de estudio, organización de planes y horarios, realización de exámenes, búsqueda de información, comunicación académica escrita y oral.

Los datos obtenidos se trasladaron al formato SPSS, para el logro del objetivo y verificación de hipótesis se empleó la Prueba Chi Cuadrado y se evaluaron asociaciones bivariantes por medio del coeficiente de correlación lineal para posteriormente realizar análisis de regresión lineal múltiple. Finalmente se emplea Análisis de Correspondencias Múltiples, para relacionar las tres variables de investigación, a través de sus puntajes categorizados.

\section{RESULTADOS}

Tabla 1. Distribución de estudiantes de la Escuela Profesional de Medicina Veterinaria de la Universidad Nacional Mayor de San Marcos, según años de estudio y género

\begin{tabular}{ccccccc}
\hline \multirow{2}{*}{ Sexo } & \multicolumn{5}{c}{ Año de Estudios } & \multirow{2}{*}{ Total } \\
\cline { 2 - 6 } & Segundo & Tercero & Cuarto & Quinto & Sexto & \\
\hline Masculino & 17 & 13 & 17 & 14 & 4 & 65 \\
Femenino & 22 & 32 & 25 & 13 & 6 & 98 \\
Total & 39 & 45 & 42 & 27 & 10 & 163 \\
\hline
\end{tabular}

De los estudiantes encuestados la mayoría; $27,61 \%$ son del tercer año de estudios, $60,12 \%$ son de género femenino, $60,12 \%$ proceden de instituciones educativas de gestión particular. El rango de edades en el género masculino está entre los 18 a 54 años, en el género femenino es de 17 a 29 años, según grupo etario y género se presentan 12,02\% estudiantes adolescentes (entre 17 a 19 años) y $84,66 \%$ estudiantes jóvenes (entre 20 a 29 años).

El $63,19 \%$ de estudiantes, han participado en actividades académicas, talleres $u$ otros sobre hábitos de estudio. El 60,74\%, responde que no ha participado en actividades académicas, talleres $u$ otros sobre motivación para el aprendizaje. El $78,53 \%$ de estudiantes responde que no ha llevado un curso sobre hábitos de estudio, como parte de su plan de estudios. La mayoría $82,71 \%$, responde que no ha asistido por cuenta propia a un taller o similar sobre hábitos de estudio en instituciones externas a la universidad.

Antes de emplear las pruebas estadísticas inferenciales verificamos la aleatoriedad de la muestra empleando la prueba no paramétrica de Rachas. (Gibbons y Chakraborti, 2003). Con un nivel de confianza $\alpha=0,05(5 \%)$, el valor del $p$ value $=0,388$ no es menor que $\alpha=0,05$, por tanto se asume que la muestra aplicada es aleatoria.

Tabla 2. Nivel de rendimiento académico (RA) y nivel de hábitos de estudio (HE) de estudiantes de la Escuela Profesional de Medicina Veterinaria de la Universidad Nacional Mayor de San Marcos

\begin{tabular}{llll}
\hline \multirow{2}{*}{ Rendimiento } & \multicolumn{2}{c}{ Hábitos de estudio } & \multirow{2}{*}{ Total } \\
\cline { 2 - 3 } & Regular Bueno Optimo & \\
\hline
\end{tabular}




\begin{tabular}{ccccc}
\hline Bajo & 4 & 10 & 1 & 15 \\
Regular & 22 & 115 & 11 & 148 \\
Total & 26 & 125 & 12 & 163 \\
\hline
\end{tabular}

Se observa que la mayoría de estudiantes, $148(90,80 \%)$ tiene nivel de rendimiento regular es decir de 11 a 14,99 de promedio ponderado. Asimismo la mayoría de estudiantes, 125 (76,69\%) tiene nivel de hábitos de estudios bueno, es decir de 27 a 39.

Para verificar la independencia entre el rendimiento académico y los hábitos de estudio se utilizó la prueba de razón de verosimilitud, con un nivel de confianza $\alpha=0,05$ (5\%), el $p$-value es 0,537 , que no es menor de 0,05 , por tanto el rendimiento académico y los hábitos de estudios son independientes, es decir no están asociados o relacionados.

Tabla 3. Prueba Chi Cuadrado para verificar la independencia entre el nivel de rendimiento académico (RA) y nivel de hábitos de estudio (HE)

\begin{tabular}{lccc}
\hline & Valor & gl & $\begin{array}{l}\text { Sig. } \\
\text { (bilateral) }\end{array}$ \\
\hline Chi-cuadrado de Pearson & $1,416^{\mathrm{a}}$ & 2 &, 493 \\
Razón de verosimilitudes & 1,245 & 2 &, 537 \\
Asociación lineal por lineal &, 947 & 1 &, 330 \\
N de casos válidos & 163 & & \\
\hline
\end{tabular}

a. 2 casillas $(33,3 \%)$ tienen una frecuencia esperada inferior a 5 . La frecuencia mínima esperada es 1,10.

Prueba de hipótesis de independencia entre nivel de rendimiento académico (RA) y nivel de hábitos de estudio (HE)

$\mathrm{H}_{0}$ : Nivel del rendimiento académico y nivel de hábitos de estudio son independientes.

$\mathbf{H}_{1}$ : Nivel del rendimiento académico y nivel de hábitos de estudio no son independientes. $\alpha=0,05(5 \%)$

Observando la Tabla 3, prueba chicuadrado, se empleó la prueba de razón de verosimilitud, dado que más del $20 \%$ de celdas tiene frecuencia esperada menor de 5 , en este caso es del 33,3\%. El p-value correspondiente es 0,537 , que no

Tabla 4. Nivel del rendimiento académico (RA) y nivel de motivación para el aprendizaje (MA) de los estudiantes de la Escuela Profesional de Medicina Veterinaria de la Universidad Nacional Mayor de San Marcos

\begin{tabular}{|c|c|c|c|c|}
\hline \multicolumn{5}{|c|}{ Nacional Mayor de San Marcos } \\
\hline \multirow[t]{2}{*}{ Rendimiento } & \multicolumn{3}{|c|}{$\begin{array}{l}\text { Nivel de motivación para el } \\
\text { aprendizaje }\end{array}$} & \multirow[t]{2}{*}{ Tota } \\
\hline & Regular & Bueno & Optimo & \\
\hline Bajo & 3 & 9 & 3 & 15 \\
\hline Regular & 14 & 87 & 47 & 148 \\
\hline Total & 17 & 95 & 50 & 163 \\
\hline
\end{tabular}

es menor de 0,05 ; por tanto no se rechaza $\mathrm{H}_{0}$. Es decir al $5 \%$ de nivel de significación se asume que el nivel del rendimiento académico y nivel de hábitos de estudios son independientes, es decir no están asociados o relacionados.

Este resultado se debe a que hay homogeneidad, dada que en la Tabla 4 se observa que el $90,80 \%$ de estudiantes tienen nivel de rendimiento regular es decir de 11 a 14,99 de promedio ponderado. Asimismo el $76,69 \%$ de estudiantes tiene nivel de hábitos de estudios bueno es decir de 27 a 39. 
Se observa que la mayoría de estudiantes, $148(90,80 \%)$ tiene nivel de rendimiento regular es decir de 11 a 14,99 de promedio ponderado. Asimismo la mayoría de estudiantes, 95 (58,28\%), tiene nivel motivación para el aprendizaje bueno es decir de 13 a 18.

Para verificar la independencia entre el rendimiento académico y la motivación para el aprendizaje se utilizó la prueba de razón de verosimilitud, con un nivel de confianza $\alpha=0,05(5 \%)$, el $p$-value es 0,393 , que no es menor de 0,05 , por tanto el rendimiento académico y la motivación para el aprendizaje son independientes, es decir no están asociados o relacionados.

Tabla 5. Prueba Chi Cuadrado para verificar la independencia entre el nivel de rendimiento académico (RA) y nivel de motivación para el aprendizaje (MA)

\begin{tabular}{lccc}
\hline & Valor & gl & $\begin{array}{c}\text { Sig. asintótica } \\
\text { (bilateral) }\end{array}$ \\
\hline Chi-cuadrado de Pearson & $2,068^{\mathrm{a}}$ & 2 &, 356 \\
Razón de verosimilitudes & 1,869 & 2 &, 393 \\
Asociación lineal por lineal & 1,819 & 1 &, 177 \\
N de casos válidos & 163 & & \\
\hline
\end{tabular}

a. 2 casillas $(33,3 \%)$ tienen una frecuencia esperada inferior a 5 . La frecuencia mínima esperada es 1,56.

Prueba de hipótesis de Independencia entre nivel de rendimiento académico y nivel de motivación para el aprendizaje.

$\mathrm{H}_{0}$ : Nivel del rendimiento académico y nivel de motivación para el aprendizaje son independientes.

$\mathbf{H}_{1}$ : Nivel del rendimiento académico y nivel de motivación para el aprendizaje no son independientes.

$\alpha=0,05$ (5\%)

Observando la Tabla 5, prueba chicuadrado, se empleara la prueba de razón de verosimilitud, dado que más del $20 \%$ de celdas tiene frecuencia esperada menor de 5, en este caso es del 33,3\%. El p-value correspondiente es 0,393 , que no es menor de 0,05 ; por tanto no se rechaza $\mathrm{H}_{0}$. Es decir al $5 \%$ de nivel de significación se asume que el nivel del rendimiento académico y nivel de motivación para el aprendizaje son independientes, es decir no están asociados o relacionados.

Este resultado se debe a que hay homogeneidad en la muestra, en la tabla 5 se observa que el $90,80 \%$ tiene nivel de rendimiento regular es decir de 11 a 14,99 de promedio ponderado. Asimismo el $58,28 \%$ tiene nivel motivación para el aprendizaje bueno, es decir de 13 a 18.

Mediante el uso del coeficiente de correlación de Pearson, se observa que la relación lineal entre el rendimiento académico los hábitos de estudio es de 0,132 , lo que indica escasa relación lineal entre ambas variables, la relación lineal entre el rendimiento académico y la motivación para el aprendizaje es 0,197, indica escasa relación lineal entre ambas variables.

Tabla 6. Prueba Chi Cuadrado para verificar la independencia entre el nivel de rendimiento académico (RA) y nivel de motivación para el aprendizaje (MA)

\begin{tabular}{lll} 
& $\begin{array}{l}\text { Promedio } \\
\end{array}$ & Motivación \\
\hline Hábitos de estudio &, 132 &, 315 \\
Motivación para el aprendizaje &, 197 & \\
\hline
\end{tabular}


En el caso de la relación lineal entre los hábitos de estudio y la motivación para el aprendizaje es 0,315, indica una escasa relación lineal entre ambas variables, por lo tanto el modelo de regresión lineal estimado se considera estadísticamente no significativo.
El coeficiente de determinación es 0,044 que indica que sólo el $4 \%$ de la variabilidad de la variable dependiente, rendimiento académico, se explica por las variables independientes hábitos de estudio y motivación para el aprendizaje.

Tabla 7. Medidas globales que evalúan el modelo de regresión lineal múltiple estimado

\begin{tabular}{lllllll}
\multicolumn{2}{l}{ Resumen del modelo } \\
\hline Modelo & $\mathrm{R}$ & $\mathrm{R}$ cuadrado & $\mathrm{R}$ corregida & Estror típ. de la \\
estimación & \\
\hline 1 &, $211^{\mathrm{a}}$ &, 044 &, 032 & 1,06642 \\
\hline
\end{tabular}

a. Variables predictoras: (Constante), Hábitos, motivación.

b. Variable dependiente: Rendimiento académico (promedio ponderado)

A fin de obtener mayor información cuantitativa y por tanto mayor conocimiento del problema de investigación se aplicó el Análisis de Correspondencias Múltiples donde se analiza desde un punto de vista gráfico, las relaciones de dependencia e independencia de un conjunto de variables categóricas, de forma que la proximidad entre los puntos representados está relacionada con el nivel de asociación entre dichas modalidades.

Las variables utilizadas hábitos de estudios (HE), motivación para el aprendizaje (MA) y rendimiento académico (RA), se categorizaron de la siguiente manera:

\begin{tabular}{cl} 
Clave & \multicolumn{1}{c}{ Niveles } \\
\hline HR & Hábito de estudio regular \\
HB & Hábito de estudio bueno \\
HO & Hábito de estudio optimo \\
MR & Motivación para el aprendizaje regular \\
MB & Motivación para el aprendizaje buena \\
MO & Motivación para el aprendizaje optima \\
RA2 & Rendimiento académico bajo \\
RA3 & Rendimiento académico regular \\
\hline
\end{tabular}

Las variables ilustrativas son: año de tipo de gestión de la institución educativa estudios, género, edad (grupo etario) y

(IE) secundaria de egreso.

\begin{tabular}{ll}
\hline Claves & Características \\
\hline 2 & Segundo año de estudios. \\
3 & Tercer año de estudios. \\
4 & Cuarto año de estudios. \\
5 & Quinto año de estudios. \\
6 & Sexto año de estudios. \\
Femn & Femenino. \\
Masc & Masculino. \\
$17-19$ & Edad de 17-19 años. \\
$20-24$ & Edad de 20-24 años. \\
$25-29$ & Edad de 25-29 años.
\end{tabular}


30 y más $\quad$ Edad de 30 a más años.

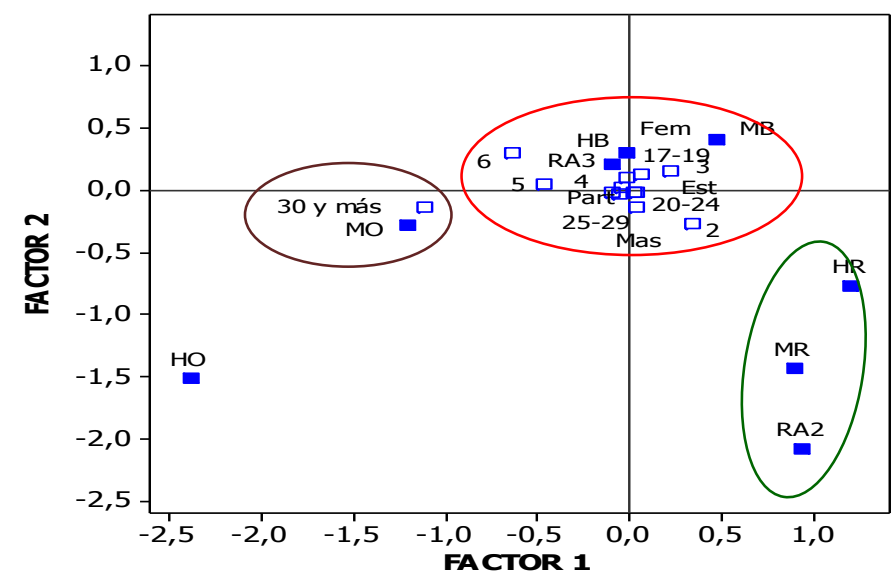

Figura 1. Análisis factorial de correspondencias múltiples: Nivel de hábitos de estudio (HA), nivel de motivación para el aprendizaje (MA) y nivel de rendimiento académico (RA).

En la Figura 1 interpretando de izquierda a derecha, el primer grupo de modalidades la motivación de nivel optima (MO) se asocia a estudiantes de 30 y más años.

El segundo grupo de modalidades la motivación de nivel buena (MB) y hábitos de estudio de nivel bueno (HB) se asocia a estudiantes con rendimiento académico regular (RA3) y no hay diferencial con años de estudio, genero, grupos de edad e Institución educativa de egreso de la secundaria.

El tercer grupo de modalidades hábitos de estudio de nivel regular (HR) y la motivación de nivel regular (MR) se asocia a estudiantes con rendimiento académico bajo (RA2).

\section{DISCUSIÓN}

No existe relación entre los hábitos de estudio y motivación para el aprendizaje con el nivel de rendimiento académico de los estudiantes de Medicina Veterinaria de la Universidad Nacional Mayor de San Marcos, el resultado nos muestra que la correlación es escasa por lo tanto no existe relación entre estas variables, se considera como una causa la homogeneidad en la muestra en relación al rendimiento académico, es decir la mayoría de estudiantes presentan rendimiento académico con un reducido rango entre 11 y 14,99, a pesar que todos no han cursado las mismas asignaturas, por otro lado más del $75 \%$ de estudiantes presentan nivel de hábitos de estudio bueno y más del $50 \%$ de estudiantes presentan nivel de motivación para el aprendizaje bueno, estamos frente a un grupo de estudiantes que presentan características muy parecidas por lo que la correlación entre las variables en estudio resultó estadísticamente no significativa.

No existe relación entre los hábitos de estudio y el rendimiento académico de los estudiantes de Medicina Veterinaria, el resultado muestra que las variables son independientes, por lo tanto no existe relación entre ellas, debido a que los estudiantes en su mayoría presentan buenos hábitos de estudio y similar rendimiento académico. En la investigación de (Villegas et al., 2009) se encontró una correlación débil entre los hábitos de estudio y el rendimiento académico, concluyéndose que en la población estudiada existen otro tipo de factores que están afectando en mayor 
grado el desempeño académico. Cabrera y Sánchez (2004), manifiestan que los hábitos de estudio no muestran relación con el desempeño académico por lo que evidencian la necesidad de impulsar o reforzar la tarea de investigación en este campo. Tomando como contraparte la investigación de (Vildoso, 2003) determinó que existe asociación significativa de los hábitos de estudio y la autoestima en el rendimiento académico de los alumnos de segundo, tercero y cuarto año de la carrera de Agronomía de la Universidad Nacional Jorge Basadre Grohmann, presentando un porcentaje considerable de alumnos con nivel bajo de hábitos de estudio y baja autoestima y la población de estudiantes muestra un nivel bajo de rendimiento académico.

No existe relación entre la motivación para el aprendizaje y el rendimiento académico de los estudiantes de Medicina Veterinaria, el resultado muestra que estas variables son independientes y por lo tanto no existe relación entre ellas, debido a que la mayoría de los estudiantes presentan motivación para el aprendizaje buena y similar rendimiento académico. En la investigación de Geukes, Rehbein, Sales y Serrano, 2007), la motivación para el aprendizaje y el nivel de rendimiento académico no están relacionados, son independientes, no hubo diferencias significativas entre la motivación y las calificaciones altas a pesar que su hipótesis esperaba una alta correlación, mencionan como un factor que contribuyó a este resultado que para obtener una variable dependiente fiable y homogénea incluyeron únicamente estudiantes de psicología cuyas notas tienen el mismo origen respecto a la evaluación del rendimiento. Sin embargo, la limitación de usar como sujetos solamente estudiantes de una carrera también puede haber disminuido la variación en las respuestas y así la probabilidad de obtener correlaciones estadísticamente significativas. Por otro lado en la investigación de Félix, (2015), concluye que la motivación debe de tomarse en consideración según su naturaleza, ya sea extrínseca o intrínseca debido a que cada tipo de motivación afecta de diferente forma al estudiante. La motivación intrínseca por su parte se muestra relacionada directamente con el rendimiento académico, por lo que se debe fomentar su desarrollo en el estudiante para incrementar el rendimiento académico; en cambio, con una motivación puramente extrínseca se corre el riesgo de que el rendimiento académico caiga, debido a que esta se refiere a la motivación que nace de los valores fuera del individuo y de la actividad de aprendizaje.

De la mayoría $82,71 \%$ de los estudiantes encuestados, responden que no han asistido por cuenta propia a un taller o similar sobre hábitos de estudio en instituciones externas a la universidad. Este resultado indica el poco interés, poca disponibilidad de tiempo o falta de información para asistir a este tipo de actividades de parte de los estudiantes.

Al analizar los datos obtenidos por año de estudios, género, edad e institución educativa de egreso de la secundaria de los estudiantes de Medicina Veterinaria, no se observaron diferencias estadísticamente significativas $(p>0,05)$, por lo que se considera que son similares, en las investigaciones de Acevedo, Torres, y Tirado, (2015) y Dominino y Castellaro, (2012) los resultados fueron similares. Por otro lado en la investigación de (Navea, 2015) sobre motivación y estrategias de aprendizaje en estudiantes universitarios de ciencias de la salud el resultado señala que las mujeres se preocupan más por no quedar mal ante los demás y presentan más ansiedad; mientras que los hombres tienen puntuaciones más elevadas en autoensalzamiento del ego, presentan más competencia y control para las tareas y el rendimiento, señala entonces que los hombres tienen una mayor motivación extrínseca que las mujeres. En relación al nivel educativo también encontró diferencias significativas concluyendo que los estudiantes según avanzan de curso están más preocupados por ser enjuiciados por los demás y quedar bien, y además están más preocupados y ansiosos una vez que ya se han situado en los estudios. Según (Neri, 2014) las mujeres están más motivadas para el aprendizaje que los hombres y buscan más un aprendizaje real, en las formas de estudiar, en ver la utilidad de las asignaturas. $\mathrm{Y}$ lo mismo sucede con la 
edad, aunque menos significativa, los más jóvenes se conforman con aprobar, mientras que los alumnos mayores y experimentados desean sacar más partido a sus estudios tratando de aprender lo máximo posible.

Al hacer un análisis estadístico más profundo encontramos que hay un grupo de estudiantes de Medicina Veterinaria que presentan hábitos de estudios regular y motivación para el aprendizaje regular y tienen rendimiento académico bajo, es decir, presentan hábitos de estudio y motivación para el aprendizaje pero que tienen que ser reforzados con las técnicas de estudio y a la vez se debe motivar al estudiante para que cambie de manera positiva sus hábitos de estudio y logre elevar su nivel de rendimiento académico. En la investigación de (Velásquez, 2014) señala que los alumnos con una motivación intermedia, que tienen buenas y malas puntuaciones presentan esta variabilidad debido a que el estar motivado es necesario pero no suficiente para obtener un buen rendimiento, y son necesarias una serie de variables más como son los conocimientos previos, las capacidades intelectuales, estilos de aprendizaje, autoconcepto para que el rendimiento sea satisfactorio. (Velásquez, 2014).

En relación al grupo de estudiantes que tienen entre 30 y más años presentan una motivación para el aprendizaje optima debido a que su motivación es fundamentalmente intrínseca, el aprendizaje adulto tiene una motivación para aprender que no se centra en el rendimiento académico. En las personas mayores, la satisfacción personal que experimentan al participar voluntariamente en un proceso de aprendizaje y culminarlo, es de especial relevancia (Cuenca, 2011). Diferentes investigaciones ponen de manifiesto que los estudiantes que adoptan una orientación motivacional intrínseca, emplean estrategias cognitivas y procesos autorregulados en mayor medida que los estudiantes que adoptan una orientación motivacional extrínseca (Cerezo y Casanova, 2004). No conocemos el grado de motivación extrínseca de nuestros estudiantes, es decir, si están motivados a obtener buenas notas para cumplir con las expectativas de las personas significativas para ellos, por ello necesitamos realizar nuevas investigaciones sobre este tema en la educación veterinaria.

El rendimiento académico, los hábitos de estudios y la motivación para el aprendizaje en los estudiantes de medicina veterinaria no están asociados o relacionados, presenta baja relación lineal frente a los hábitos de estudio y la motivación para el aprendizaje.

Los estudiantes de medicina veterinaria de 30 y más años presentan una motivación para el aprendizaje óptima; y aquellos que presentan hábitos de estudio y motivación para el aprendizaje bueno tienen rendimiento académico regular. Los estudiantes de medicina veterinaria que presentan hábitos de estudio y motivación para el aprendizaje regular tienen rendimiento académico bajo.

\section{REFERENCIAS BIBLIOGRAFICAS}

Acevedo, D., Torres, J. D., \& Tirado, D. F. (2015). Análisis de los Hábitos de Estudio y Motivación para el Aprendizaje a Distancia en Alumnos de Ingeniería de Sistemas de la Universidad de Cartagena (Colombia). Formación universitaria, 8(5), 59-66.

Cabrera, A. M. A., \& Sánchez, A. W. M. (2004). Hábitos de estudio y rendimiento académico. México: Universidad de Guanajuato.

Cerezo Rusillo, M., \& Casanova Arias, P. F. (2004). Diferencias de género en la motivación académica de los alumnos de Educación Secundaria Obligatoria. Electronic journal of research in educational psychology, 2(1), 97-112.

Cuenca, M. E. (2011). Motivación hacía el aprendizaje en las personas mayores más allá de los resultados y el rendimiento académico. Revista de Psicología de la Educación, 6, 171186.

Dominino, M., \& Castellaro, M. A. (2012). RELACIÓN ENTRE HÁBITOS DE ESTUDIO Y VARIABLES SOCIOACADÉMICAS ESPECÍFICAS EN ESTUDIANTES DE PSICOLOGÍA. In IV Congreso Internacional de 
Investigación y Práctica Profesional en Psicología XIX Jornadas de Investigación VIII Encuentro de Investigadores en Psicología del MERCOSUR. Facultad de PsicologíaUniversidad de Buenos Aires.

Félix, A. (2015). Influencia de la motivación en el rendimiento académico de primer año de los alumnos de las carreras de ingeniería en sistemas computacionales, ciencias de la comunicación, administración de empresas y gastronomía de una universidad privada de México. Tesis para optar el grado de maestría en Educación con acentuación en desarrollo cognitivo. Tecnológico de Monterrey. Culiacán, Sinaloa, México.

Gibbons, J. D., \& Chakraborti, S. (2003). Nonparametric statistical inference fourth edition, revised and expanded. STATISTICS TEXTBOOKS AND MONOGRAPHS, 168.

Geukes, S., Rehbein, M., Sales, M., Serrano, V., (2007). Relación entre motivación y rendimiento académico. pp. 18. Alemania. Recuperado de

http://

www.ucm.es/info/teamwork/abarras a/tea/200607 26921/22-1.pdf

Navea, A. (2015). Un estudio sobre la motivación y estrategias de aprendizaje en estudiantes universitarios de ciencias de la salud. Tesis para optar el grado de Doctor. Universidad Nacional de Educación a Distancia UNED, España.

Neri, I. (2014). Aprobar o aprender, ¿qué busca el alumnado en la formación profesional? Tesis para optar el grado de Master universitario en formación del profesorado de educación secundaria. Universidad Pública de Navarra, España.

Oñate, C. \& Valiente, M. (Mayo, 1991). Los hábitos de estudio y la motivación para el aprendizaje en los alumnos de primer curso de la Escuela de Arquitectura Técnica de la Universidad Politécnica de Madrid y la incidencia en la calidad de la enseñanza. Ponencia presentada en el Congreso sobre Calidad de la Educación Universitaria, Puerto de Santa María, España.

Pola, A. H. (2013). Relación entre el rendimiento académico y hábitos de estudio en alumnos universitarios. Tesis de grado, facultad de Psicología. Universidad Abierta Interamericana, Argentina.

San Martín, H., \& García, P. (2002). Educación veterinaria en el Perú. Revista de Investigaciones Veterinarias del Perú, 13(1), 98103.

Velásquez, C. (2014). La motivación extrínseca y su relación con el rendimiento académico en el idioma inglés en los estudiantes de nivel secundaria turno tarde de la institución educativa emblemática Elvira García y García Pueblo Libre, Lima, 2014. Tesis para optar el título de licenciado en educación. Universidad Nacional de Educación Enrique Guzmán y Valle, Lima.

Vildoso, V. (2003). Influencia de los Hábitos de Estudio y la Autoestima en el Rendimiento Académico de los Estudiantes Universitarios de la Escuela Profesional de Agronomía de la Universidad Nacional Jorge Basadre Grohmann. Tesis para optar el grado académico de Magíster en Educación, Universidad Nacional Mayor de San Marcos, Lima.

Villegas-Osuna, C. A., Muñoz-Osuna, F. O., \& Villegas-Osuna, R. E. (2009). Hábitos de estudio de los alumnos en el área de Química Orgánica y su impacto en el rendimiento académico. Biotecnia, 11(3), 33-43.

Correo

electrónico:

gracielaiya@hotmail.com

Revisión de pares:

Recibido:

Aceptado: 